\title{
Melanoma metastásico con tumor primario oculto*
}

\author{
Drs. PABLO BÓRQUEZ M. ${ }^{1}$, GABRIELA OCHOA S. ${ }^{2}$, MAURICIO CORREA D. ${ }^{2}$ \\ 1 Servicio de Cirugía Hospital Base de Osorno, Campo Clínico Osorno, Facultad de Medicina, Universidad Austral \\ de Chile. \\ 2 Alumno Facultad de Medicina, Universidad Austral de Chile. \\ Osorno, Chile.
}

\begin{abstract}
\section{Metastatic melanoma with occult primary tumor}

In $3 \%$ of melanomas, the primary tumor is occult. We report a 58 years old female presenting with rapidly growing painless left inguinal lymphadenopathies. The patient was reassessed two months later, verifying that the lesions enlarged further. A CAT scan of the left inguinal region showed a solid mass in the subcutaneous of the proximal region of the left thigh. An incisional biopsy of the inguinal mass was performed. The pathological study disclosed malignant melanoma. The patient was subjected to an iliofemoral lymphatic excision and to five sessions of regional radiotherapy. After two years of follow up, the patient is without evidence of tumor recurrence.
\end{abstract}

Key words: Melanoma, metastasis, lymphadenopathies.

\section{Resumen}

En el 3\% de los melanomas, el tumor primario está oculto. Reportamos una mujer de 58 años, que presentaba linfadenopatías inguinales dolorosas. Reevaluada dos meses después, se constató crecimiento de la masa. Una TAC motró una masa sólida subcutánea en zona proximal de muslo izquierdo, la que fue biopsiada, demostrándose un melanoma. Se realizó disección linfática ilofemoral y cinco sesiones de radioterapia. A los dos años, la paciente no evidencia recurrencia tumoral.

Palabras clave: Melanoma oculto, metástasis, linfadenopatías.

\section{Introducción}

El melanoma metastásico con tumor primario oculto (MPO) corresponde a un fenómeno de metástasis sin una lesión primaria identificable. Debe ser confirmado histopatológicamente y requiere una evaluación clínica e imagenológica completa ${ }^{1-6}$. La etiología de MPO es desconocida y se han sugerido múltiples hipótesis, siendo la más apoyada la regresión espontánea del tumor primario mediada por mecanismos inmunes ${ }^{7}$. El primer paciente con MPO fue registrado en 1917, tras la revisión de una serie

*Recibido el 25 de abril de 2011 y aceptado para publicación el 15 de agosto de 2011.

Correspondencia: Dr. Pablo Bórquez M.

Av. Guillermo Bühler 1712, Osorno, Chile. Fax: 56-064-253733.

cir.oncologo@yahoo.es 
de $\operatorname{casos}^{8}$, sin embargo, la primera caracterización de los criterios para MPO fueron establecidos en $1963^{1}$. Una reciente revisión sistemática reportó 4.348 casos de MPO en 132.643 pacientes con melanoma estableciendo una frecuencia de $3,2 \%{ }^{2}$. Los diversos estudios mostrarían una conducta biológica diferente para el melanoma tradicional o de primario conocido y los MPO en cuanto a supervivencia, respuesta a intervenciones terapéuticas y factores pronósticos ${ }^{1-6,9}$. El objeto del trabajo es reportar un caso de MPO con metástasis a linfonodos ilíacos e inguinales estudiado y tratado en Hospital Base de Osorno.

\section{Caso clínico}

Paciente de 58 años, portadora de HTA y dislipidemia, en tratamiento con enalapril (10 mg c/12 hr), aspirina (100 mg/día) y atorvastatina (10 mg/ día). Consulta en el consultorio externo de cirugía del Hospital San José de Osorno, derivada desde policlínico periférico, con diagnóstico de conglomerado de adenopatías palpables de la región inguinal izquierda, de 3 meses de evolución con crecimiento rápido y no doloroso. Trae Ecografía de partes blandas que muestra imágenes nodulares de región inguinal izquierda y raíz del muslo de 60 x 30 mm con aspecto de conglomerado de adenopatías de etiología incierta. De sus exámenes, destacan LDH de 455, VHS de 20 y PCR negativa. Se considera portadora de un cuadro inflamatorio, dejándose tratamiento médico y control posterior para reevaluar necesidad de biopsia. Se controla 2 meses después y clínicamente se constata un mayor aumento de volumen inguinal, además trae un TAC solicitado por médico del extrasistema (Figura 1), el cual muestra un proceso expansivo sólido e irregular del tejido subcutáneo de 53 x 34 x 36 mm, ubicado en muslo proximal izquierdo, que por su aspecto sugiere un sarcoma de partes blandas, además describe adenopatías vecinas al trayecto de vena safena interna (la mayor de $18 \mathrm{~mm}$ ). Se ingresa al servicio de cirugía para realizar biopsia.

Se realiza una biopsia excisional de una masa inguinocrural de 8 x $5 \mathrm{~cm}$, compuesta por linfonodos aumentados de tamaño y consistencia y aspecto de presentar necrosis. Diagnóstico postoperatorio sospecha de Linfoma. Por aspecto de las lesiones y exámenes previos se decide etapificar como linfoma resultando HTLV I y II negativos, B2 microglobulina negativa, LDH normal y VHS de $36 \mathrm{~mm}$, TAC de tórax, abdomen y pelvis con contraste son normales. TAC de cuello con contraste muestra sólo un pequeño nódulo tiroideo inespecífico de $9 \mathrm{~mm}$.

El informe de la pieza quirúrgica dice "fragmento ovoide de 85 x 45 x $30 \mathrm{~mm}$, adiposo y congestivo, de contornos nodulares, posiblemente tumoral, extensamente necrótico y hemorrágico”, el estudio histológico informa tumor maligno poco diferenciado altamente sugerente de melanoma maligno. Tinción de Fontana Masson para melanina (+).

Frente a este hallazgo se reevalúa el caso en consultorio de oncología. Se reexamina la paciente (incluyendo la vía aerodigestiva superior) no encontrando el tumor primario. Sólo se encontró un pequeño nevus sospechoso en la mama derecha que se extirpa, la biopsia informa nevus compuesto.

Se realiza el siguiente estudio:

- Evaluación coloproctológica/colonoscopia, informa sólo diverticulosis extensa de colon izquierdo y hemorroides mixtos, descartando un melanoma.

- Evaluación ginecológica, que no muestra lesiones pigmentadas.

- Evaluación oftalmológica, que no muestra lesiones sugerentes de melanoma.

El comité oncológico del Hospital de Osorno solicita un PET/SCAN (Figuras 2 y 3), el que informa: adenopatía ilíaca externa de $34 \mathrm{~mm}$, otra inguinal de $17 \mathrm{~mm}$ y otra en ilíaca común izquierda de $10 \mathrm{~mm}$, con aspecto de corresponder a localizaciones secundarias. El PET confirma 2 focos ávidos de glucosa en correspondencia con adenopatías de cadena ilíaca externa e inguinal izquierda (las de 34 y $17 \mathrm{~mm}$ ) compatibles con localizaciones secundarias.

Se realiza disección linfática iliofemoral izquierda resecándose varias adenopatías inguinales e ilíacas externa hasta la bifurcación con ilíaca interna, en la exploración hacia proximal no se aprecian otras adenopatías y la resección se consideró $\mathrm{R}_{\mathrm{o}}$ (Figuras 4 y 5). La paciente es dada de alta al $5^{\circ}$ día postoperatorio, sin drenaje y evoluciona con un linfocele que se infecta (cultivo $(+)$ a pseudomona ae. sensible a

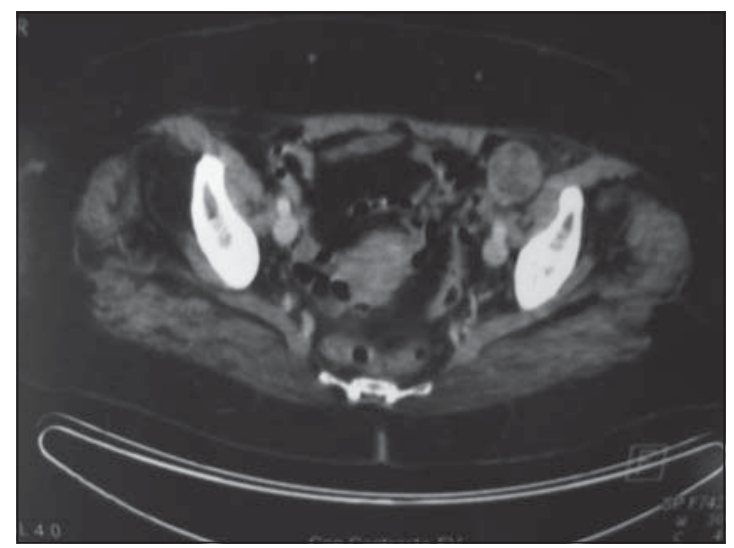

Figura 1. Imagen de TAC que muestra conglomerado de adenopatías metastásicas ileofemoral izquierda de melanoma de $34 \mathrm{~mm}$. 
ciprofloxacino), pero que mejora al cabo de un mes con curaciones.

La biopsia de la pieza postoperatoria informa ganglios inguinales superficiales (masa de $32 \mathrm{~mm}$ ), ganglio de Cloquet (masa de 5,2 cm) y adenopatía ilíaca de 4,5 cm, todas con metástasis ganglionar linfática de melanoma.

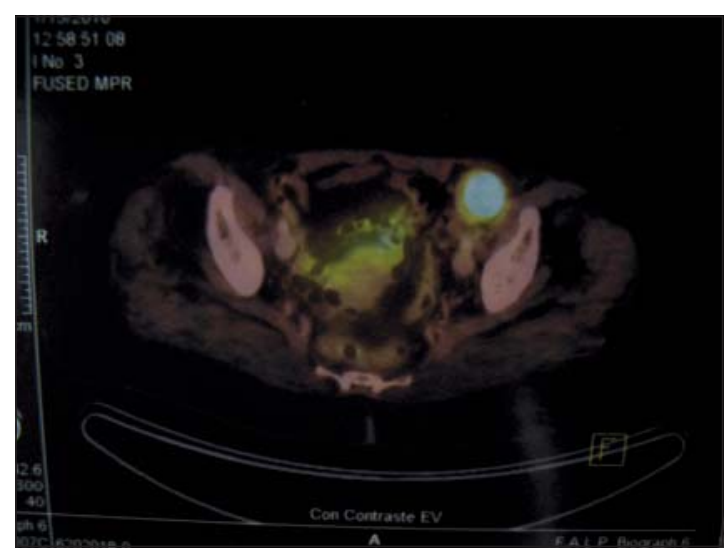

Figura 2. Imagen de PET/CT que muestra intensa captación del medio de contraste en conglomerado de adenopatías metastásicas ileofemoral izquierda de melanoma.

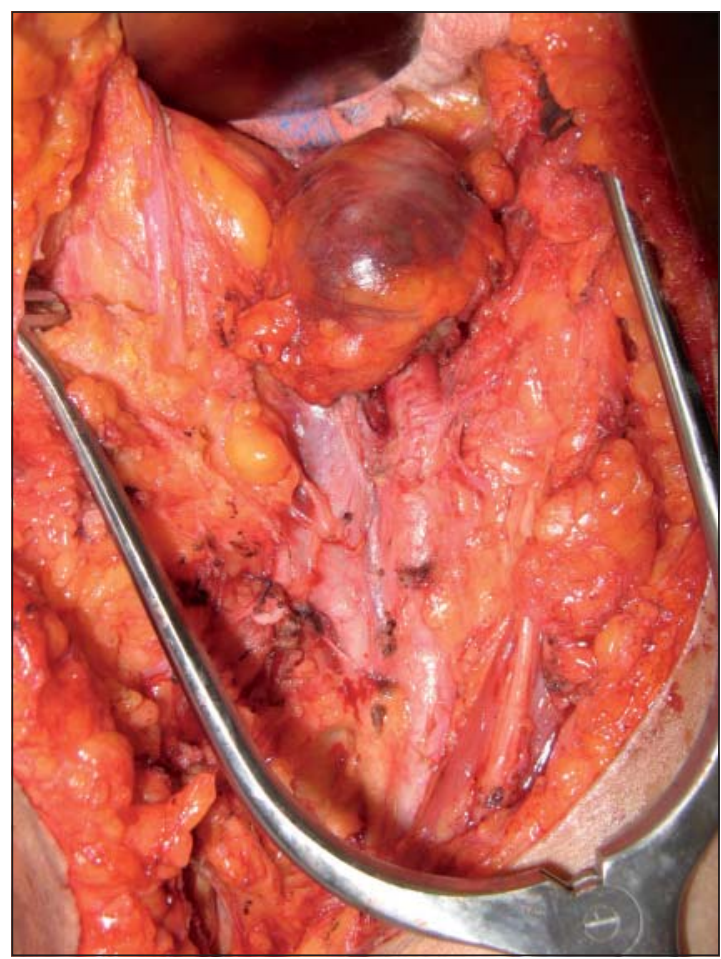

Figura 4. Imagen intraoperatoria in situ de conglomerado de adenopatías metastásicas ilíacas izquierda de melanoma. Hacia caudal de la masa se aprecian vena y arteria ilíaca.

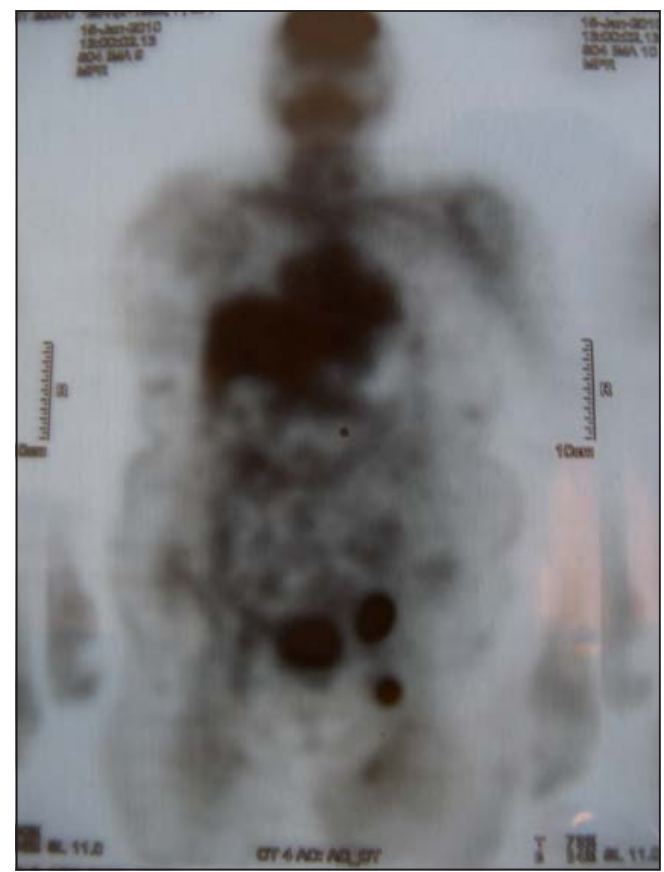

Figura 3. Imagen de PET que muestra captación del medio de contraste en conglomerado de adenopatías metastásicas ileofemoral izquierda de melanoma.

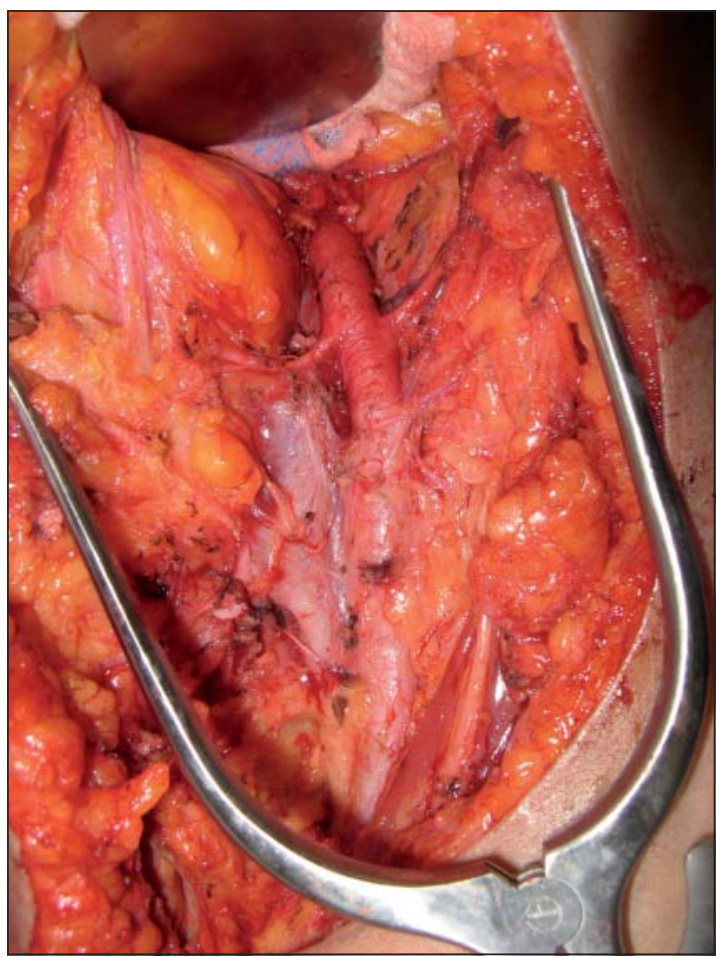

Figura 5. Imagen intraoperatoria después de realizada la disección ileofemoral. Se consideró una resección Ro. 
La paciente completa su tratamiento en Hospital Regional de Valdivia, donde recibió 30Gy en 5 fracciones (2 veces por semana) con buena tolerancia, piel con epitelitis seca grado I, sin linfedema y sin signos de recurrencia local. Dos años después la paciente se encuentra en seguimiento, sin evidencia de enfermedad.

\section{Discusión}

Los cánceres con tumor primario desconocido constituyen un reto, tanto en su diagnóstico como en su tratamiento. Inicialmente requieren de un estudio exhaustivo en busca de la lesión que le dió origen. Este grupo particular de tumores se caracterizan por presentar diseminación temprana, ausencia de clínica del tumor primario y un patrón impredecible de comportamiento tanto en agresividad como en la diseminación, por lo cual el curso clínico es incierto. En general, los pacientes con un cáncer primario oculto tienen una expectativa de vida limitada de algunos meses, sin embargo, algunos subgrupos presentan mejor pronóstico. Para establecer estos subgrupos se deben analizar algunos parámetros clinicopatológicos que incluyen la edad, género, estado funcional, pérdida de peso, histopatología, carga tumoral, localización del tumor, el número de metástasis, los sitios de origen del primario y los marcadores tumorales ${ }^{5}$.

Los melanomas en particular se presentan en $3,2 \%$ de los casos como un primario oculto, son dos veces más frecuentes en hombres y tienen un incremento en su frecuencia durante la cuarta a quinta década de la vida, representando un fenómeno aún no bien comprendido. En caso de diseminación el cuadro clínico estará relacionado a la producción de citoquinas (fiebre, pérdida de peso y/o anemia) y al sitio afectado (ictericia, derrame pleural, hepatomegalia, etc). Los sitios más frecuentes de metástasis son los linfonodos axilares, cervicales e inguinales ${ }^{2}$.

Existen algunas teorías en cuanto a su presentación dentro de las cuales una de las más aceptadas es la melanosis, que consiste en una regresión completa de la lesión maligna, con ciertas características clinicopatológicas evidenciables. Ha sido descrita en presencia de melanomas con primario conocido donde concomitantemente hay una variedad de lesiones pigmentadas o hipopigmentadas, planas, con una imagen histológica de fibrosis dérmica papilar focal y un infiltrado variable de melanófagos y linfocitos. Las células residuales de melanoma se encuentran en la periferia de la fibrosis ya sea en la epidermis, dermis o ambas. Ocasionalmente ocurre este mismo proceso en neoplasias epiteliales, carcinomas de células basales, nevus benignos y micosis fungoi- des $^{10}$. Microscópicamente son lesiones nodulares de colecciones de melanófagos CD68 (+) y marcadores melanocíticos negativos (S100, HMB-45, MelanA) ubicados principalmente en nódulos linfáticos, pudiendo existir también como melanosis tumoral ${ }^{3}$. Debido a la identidad desconocida de la neoplasia inicial, la importancia biológica y el valor clínico de la melanosis tumoral es incierto. La etiología de la melanosis aún no se conoce, sin embargo, se cree, existirían factores inmunológicos que mediarían esta regresión, adquiriendo importancia en el pronóstico $\mathrm{y}$ en el estudio de tratamientos a futuro. Raramente la regresión de un melanoma se presenta como una pápula o nódulo pigmentado biopsiable que permita descartar un proceso maligno; en cuanto a la regresión parcial ha sido reportada en 9 a 46\% de los casos de melanoma primario conocido, lo cual es indicativo de la viabilidad de esta hipótesis, sin embargo, no se puede extrapolar como regla general ${ }^{3}$.

La aproximación diagnóstica de estas lesiones requiere la confirmación histopatológica, estableciendo a qué tipo de lesión corresponde con el análisis de las muestras correspondientes tanto con microscopia de luz como inmunohistoquímica para la identificación del tipo neoplásico (S-100, HMB-45, Vimentin). Es necesaria además la historia clínica detallada y examen físico exhaustivo, que comprenda la totalidad de la piel y zonas subungueales, incluyendo además un examen oftalmológico y de mucosas oral y nasal. En caso que exista metástasis inguinales puede considerarse el realizar una colonoscopia, examen ginecológico y genital masculino. El estudio radiológico con tomografía computarizada de cabeza, cuello, cerebro en caso de síntomas (preferentemente RM), tórax, abdomen y pelvis según sea el caso puede establecerse como de rutina para el estudio de las metástasis. El rol del $\mathrm{PET} / \mathrm{TC}$ se encuentra en un proceso de evaluación y es cada vez más usado en el manejo del melanoma. Sin embargo, debido a dificultades de costo y acceso, sólo se recomienda su uso cuando los exámenes previos son no concluyentes y la resección quirúrgica es una opción razonable ${ }^{11}$. Pueden incluirse marcadores tumorales clásicos, para descartar un proceso neoplásico paralelo ${ }^{5}$.

En el caso de pacientes con MPO y metástasis a linfonodos regionales, Chang et al, reportó una supervivencia a 5 años de $55 \%$ en pacientes que se realizó una linfadenectomía, frente a una supervivencia a 5 años de $27 \%$ en pacientes sin linfadenectomía ${ }^{12}$. Por otra parte, Lee et al, estableció una supervivencia a 5 años en pacientes con MPO y melanoma con primario conocido tras linfadenectomía de $58 \pm 7 \%$ y $40 \pm 7 \%$ respectivamente 6 . Una respuesta inmune endógena contra el melanoma puede explicar la mejor tasa de supervivencia y resultados quirúrgicos 
en MPO. De igual manera justifica que no existan diferencias significativas en la utilidad de la terapia adyuvante, ya que esta podría alterar la respuesta inmunológica ${ }^{3}$. Tanto el uso de quimioterapia como radioterapia requieren de mayor estudio pero los resultados no son buenos. Para los pacientes con MPO y metástasis visceral los resultados son menos alentadores, son pacientes que tienen una sobrevida a 5 años entre el 5,9 y 18\%. No hay sobrevida a 10 años. Tampoco existe consenso en la forma de manejo de estos pacientes ${ }^{2}$.

Los factores pronósticos favorables más importantes son la regresión tumoral, un reducido número de linfonodos comprometidos y la posibilidad de realizar un manejo quirúrgico.

El enfrentamiento de un paciente con MPO requiere de un gran esfuerzo diagnóstico, requiriendo una batería de exámenes imagenológicos e histológicos completos. La etapificación de los pacientes con MPO se encuentra en discusión, debido a una mayor sobrevida comparado a pacientes portadores de melanoma tradicional. Existe consenso en el manejo quirúrgico de las lesiones cuando comprometen los linfonodos, tal como lo realizado con nuestra paciente.

\section{Referencias}

1. Das Gupta T, Bowden L, Berg J. Malignant melanoma of unknown primary origin. Surg Gynecol Obstet. 1963;117:341-5.

2. Kamposioras K, Pentheroudakis G, Pectasides D, Pavlidis N. Malignant melanoma of unknown primary site. To make the long story short. A systematic review of the literature. Crit Rev Oncol Hematol. 2010, disponible en http://www.ncbi.nlm.nih.gov/pubmed/20570171, consultado el 02/01/2011.

3. Lee C, Faries M, Wanek L, Morton D. Improved Survival After Lymphadenectomy for Nodal Metastasis From an Unknown Primary Melanoma. Clin Oncol. 2008;26:535-41.

4. Cormier J, Xing Y, Feng L, Huang X, Davidson L, Gershenwald J, et al. Metastatic Melanoma to Lymph Nodes in Patients with Unknown Primary Sites. Cancer 2006;106:2012-20.

5. Pavlidis N, Briasoulis E, Hainsworth J,Greco F. Diagnostic and therapeutic management of cancer of an unknown primary. Eur J Cancer 2003;33:1990-2005.

6. Lee C, Faries M, Wanek L, Morton D. Improved Survival for Stage IV Melanoma From an Unknown Primary Site. J Clin Oncol. 2009;27:3489-95.

7. Blessing $\mathrm{K}$, McLaren $\mathrm{K}$. Histological regression in primary cutaneous melanoma: recognition, prevalence and significance. Histopathology 1992;20:315-22.

8. Pack G, Gerber D, Scharnagel I. End results in the treatment of malignant melanoma; a report of 1190 cases. Ann Surg. 1952;136:905-11.

9. Katz K, Jonasch E, Hodi F, Soiffer R, Kwitkiwski K, Sober A, et al. Melanoma of unknown primary: experience at Massachusetts General Hospital and DanaFarber Cancer Institute. Melanoma Res. 2005;15:77-82.

10. Malafronte P, Sorrells T. Lymph Node Melanosis in a Patient with metastatic melanoma of unknown primary. Arch Pathol Lab Med. 2009;8:1332-4.

11. Mohr P, Eggermont AM, Hauschild A, Buzaid A. Staging of cutaneous melanoma. Ann Oncol. 2009;20 Suppl 6:14-21.

12. Chang AE, Karnell LH, Menck HR. The National Cancer Data Base report on cutaneous and noncutaneous melanoma: a summary of 84.836 cases from the past decade. Cancer 1998; 83:1664-78. 